\title{
Latex Agglutination Test for Early Detection of Causative Organism in Acute Bacterial Meningitis
}

\author{
Mishra B' ${ }^{1}$ Mahaseth $C^{2}$, Rayamajhi A $^{3}$ \\ ${ }^{1}$ Dr. Baliram Mishra, MBBS, DCH, MD. MPH \\ (Paediatrics), Head of department of Paediatrics, \\ Janakpur Zonal Hospital, Janakpur, ${ }^{2}$ Dr. Chandeshwar \\ Mahaseth, Professor and Chief Consultant, Kanti \\ Children Hospital, National Academy of Medical \\ Sciences (NAMS), Kathmandu, ${ }^{3}$ Dr. Ajit Rayamajhi, \\ Associate Professor, Kanti Children Hospital, National \\ Academy of Medical Sciences (NAMS), Kathmandu, \\ Nepal.
}

\section{Introduction}

A cute bacterial meningitis (ABM) is a major public health problem. It is one of the most important causes of mortality and morbidity in children. ABM accounts for an estimated annual 5, 00,000 cases worldwide with at least 50,000 deaths and an equal number of neurological disabilities ${ }^{1}$. Even with best of care and antibiotic therapy, case fatality rates remain at $5 \%$ to $10 \%$ in industrialized countries and can reach up to $20 \%$ in developing countries. Between $10 \%$ and $30 \%$ of survivors develop permanent neurological sequelae such as paralysis, epilepsy, cognitive deficit or sensorineural deafness ${ }^{2}$.

Identification of the causative organism in $A B M$ is crucial to its management as it influences the choice of antibiotics, duration of therapy as well as the disease outcome. Three organisms namely Neisseria meningitidis, Streptococcus pneumoniae and Hemophilus influenza account for more than $80 \%$ of cases of $\mathrm{ABM}^{3}$. The diagnosis of meningitis in a suspected case is based on the analysis of cerebrospinal fluid (CSF) obtained by performing a lumbar puncture ${ }^{4}$. The CSF is examined for turbidity, white blood cell (WBC) count, Gram stain and the amount of protein and sugar. Bacterial culture of the CSF has long been the gold standard for the diagnosis of ABM, but due to its poor yield, longer time and unavailability in a resource limited country like ours we decided to conduct this

\begin{abstract}
Introduction: Acute bacterial meningitis is one of the leading causes of mortality and morbidity in children. Identification of the causative organism is crucial to its management and outcome. The objective of this study was to see the usefulness of latexagglutination test in the early diagnosis of acute bacterial meningitis. Materials and Methods: A hospital based prospective cross-sectional study was conducted at Kanti Children's Hospital during December 2004 to August 2005. Cerebrospinal fluid from 150 consecutive clinically suspected cases of acute bacterial meningitis between the age group of 2 months to 14 years were analyzed. Bacterial culture and latex agglutination test was done on cerebrospinal fluid obtained from all 150 suspected cases of acute bacterial meningitis. Latex agglutination test was done using the BD Directigen $^{\mathrm{TM}}$ Meningitis Combo test kit (Becton, Dickinson and company, USA) for Streptococcus pneumoniae, group B Streptococcus, Escherichia coli, Neisseria meningitidis group $A, C$ and $Y /$ W135, and Hemophilus influenzae type b. Data was analysed by using SPSS Version 11.5. Results: Of the 150 Cerebrospinal fluid samples analysed bacterial culture identified only 4 meningitis cases giving an isolation rate of $1.3 \%$ whereas latex agglutination test identified 29 cases giving an isolation rate of $19.3 \%$ from 150 samples. Streptococcus pneumoniae, Hemophilus influenzae type $b$ and Group B Streptococcus were the most common causative organism. Conclusion: Latex agglutination test has a better yield, higher sensitivity, provides microbiological diagnosis earlier than the traditional cerebrospinal fluid culture and is easy to perform.
\end{abstract}

Key words: Acute bacterial meningitis, Cerebrospinal fluid, Latex agglutination test.

study to see the usefulness of latex agglutination test (LAT) in the early diagnosis of ABM.

\author{
Address for correspondence \\ Dr. Baliram Mishra \\ Head of Department of Paediatrics \\ Janakpur Zonal Hospital \\ Janakpur, Nepal \\ E-mail: balirammishra@yahoo.com
}

\section{How to cite this article?}

Mishra B, Mahaseth C, Rayamajhi A. Latex Agglutination Test for Early Detection of Causative Organism in Acute Bacterial Meningitis. J Nepal Paediatr Soc 2013;33(1):34-38. 
LAT relies on the detection of soluble antigen in the CSF. Pre-treated latex beads agglutinate in the presence of the bacterial antigen, allowing rapid visualization of positive agglutination to the naked eye. In contrast to the hours spent on bacterial culture LAT can be completed in approximately $20 \mathrm{~min}$ (including the time taken to heat and centrifuge the CSF sample). The LAT can differentiate between $N$. meningitidis serogroups $A, C$ and Y/W135, N. meningitidis serogroups B/Escherichia coli, Hemophilus influenzae, Streptococcus pneumoniae and Group B Streptococcus hence allowing prompt institution of directed therapy that is specific for the bacteria detected.

\section{Materials and Methods}

Study site and study population. A hospital based prospective cross-sectional study was conducted at Kanti Children's Hospital during December 2004 to August 2005. This government hospital is situated in Kathmandu and is the largest children's hospital in the country providing multispecialty affordable care to children from all over the country. All children between 2 months to 14 years of age who were a suspected case of ABM were enrolled into the study. The study was approved by the Institutional Research Board of National Academy of Medical Sciences and ethical clearance granted.

Inclusion and exclusion criteria. All children between the age of 2 months to 14 years who fulfilled the case definition of a suspected case of ABM and had no exclusion criteria were included in the study. Children in whom lumbar puncture was contraindicated or refused; those receiving immunosuppressant's; those with a history of having undergone a neurosurgical procedure and those who had received parenteral antibiotics for more than 72 hours were excluded from the study.

\section{Case definitions}

Suspected case. A child between 2 months to 14 years of age presenting with clinical features of meningitis namely fever and/or headache and/or neck stiffness and/or bulging fontanel and/or altered sensorium and/or convulsions.

Confirmed case. A suspected case whose CSF has at least one of the following findings - (1) elevated protein to more than $40 \mathrm{mg} / \mathrm{dl}$, (2) decreased glucose to less than $40 \mathrm{mg} / \mathrm{dl}$, (3) a CSF to serum glucose ratio of less than 0.6, (4) a total leukocyte count of more than 10 cells $/ \mathrm{mm}^{3}$ with neutrophillic predominance, (5) organism in Gram's stain or bacterial culture.

Laboratory methods and data analysis. After obtaining written consent lumbar puncture was done using sterile technique (thorough cleaning of local part using povidone iodine and then alcohol skin preparation; sterile disposable needle and collection tubes). Two millilitres of CSF was collected in three different clear sterile plastic collection tubes and immediately transported to the laboratory for analysis of cells, sugar, protein, Gram's stain, bacterial culture and LAT. For bacterial culture $20 \mu \mathrm{L}$ of CSF was inoculated into plates of blood agar and chocolate agar and incubated. All plates negative for growth after 48 hours were considered culture negative and discarded. LAT was done using the BD Directigen ${ }^{\mathrm{TM}}$ Meningitis Combo test kit (Becton, Dickinson and company, USA) for Streptococcus pneumoniae, group B Streptococcus, Escherichia coli, Neisseria meningitidis group A,C and Y/W135, and Hemophilus influenzae type b. CSF of all suspected cases enrolled in the study underwent bacterial culture and LAT. Data was analysed by using SPSS Version 11.5. Sensitivity, Specificity, Positive and Negative predictive values were calculated. Fischer's exact test and chi square test with Yate's correction were performed to assess statistical significance of relationship between variables and meningitis. A ' $p$ ' value of $<0.05$ was considered significant.

\section{Results}

CSF from 150 consecutive clinically suspected cases of ABM between the age group of 2 months to 14 years were analyzed. Bacterial culture and LAT was done on CSF obtained from all 150 suspected cases of ABM. 38 (25.3\%) patients were confirmed cases of $A B M$ as per the case definition. Of the 150 CSF samples analysed organisms could be identified in 29 cases as shown in Figure 1.

Table 1: Organisms identified by CSF culture and LAT

\begin{tabular}{|l|c|c|}
\hline Organisms & LAT & $\begin{array}{c}\text { CSF bacterial } \\
\text { culture }\end{array}$ \\
\hline Streptococcus pneumoniae & 12 & 2 \\
\hline Hemophilus influenzae type b & 12 & 0 \\
\hline Group B Streptococcus & 5 & 2 \\
\hline Neisseria meningitidis & 0 & 0 \\
\hline Escherichia coli & 0 & 0 \\
\hline Total & $\mathbf{2 9}$ & $\mathbf{4}$ \\
\hline
\end{tabular}

As shown in table 1 bacterial culture identified only four meningitis cases giving an isolation rate of $1.3 \%$ from 150 samples. Of the four organism identified two were Streptococcus pneumoniae and two Group $B$ Streptococcus. LAT identified 29 cases giving an isolation rate of $19.3 \%$ from 150 samples. Of the 29 organisms identified by LAT 12 (41.4\%) were Streptococcus pneumoniae, 12 (41.4\%) Hemophilus influenzae type $b$ and $5(17.2 \%)$ Group B Streptococcus. The sensitivity, specificity, positive and negative predictive values of bacterial culture and LAT has been shown in table 2,3,4. 
Table 2: Performance of Latex agglutination test

\begin{tabular}{|c|c|c|c|c|}
\hline \multirow{2}{*}{ Latex agglutination test } & \multicolumn{2}{|c|}{ Suspected case of meningitis (150) } & \multirow{2}{*}{ Total } & \multirow{2}{*}{ 'p' value } \\
\cline { 2 - 4 } & Confirmed case (38) & No meningitis (112) & & \\
\hline Positive & 23 & 6 & 129 & \multirow{2}{*}{0.0001} \\
\hline Negative & 15 & 106 & $\mathbf{1 5 0}$ & \\
\hline Total & $\mathbf{3 8}$ & $\mathbf{1 1 2}$ & \\
\hline
\end{tabular}

Table 3: Performance of bacterial culture of cerebrospinal fluid.

\begin{tabular}{|c|c|c|c|c|}
\hline \multirow{2}{*}{ CSF culture } & \multicolumn{2}{|c|}{ Suspected case of meningitis (150) } & \multirow{2}{*}{ Total } & \multirow{2}{*}{ ' $p$ ' value } \\
\cline { 2 - 4 } & Confirmed case (38) & No meningitis (112) & 4 & \multirow{2}{*}{0.0001} \\
\hline Positive & 4 & 0 & 146 & \\
\hline Negative & 34 & 112 & $\mathbf{1 5 0}$ & \\
\hline Total & $\mathbf{3 8}$ & $\mathbf{1 1 2}$ & \\
\hline
\end{tabular}

Table 4: Comparison of performance of CSF culture and Latex agglutination test.

\begin{tabular}{|l|c|c|}
\hline Test characteristics & Latex agglutination test & CSF culture \\
\hline Sensitivity & $60.5 \%$ & $10.5 \%$ \\
\hline Specificity & $94.6 \%$ & $100 \%$ \\
\hline Positive Predictive Value & $79.3 \%$ & $100 \%$ \\
\hline Negative Predictive Value & $87.6 \%$ & $16.4 \%$ \\
\hline
\end{tabular}

\section{Causative organisms of Acute Bacterial Meningitis}

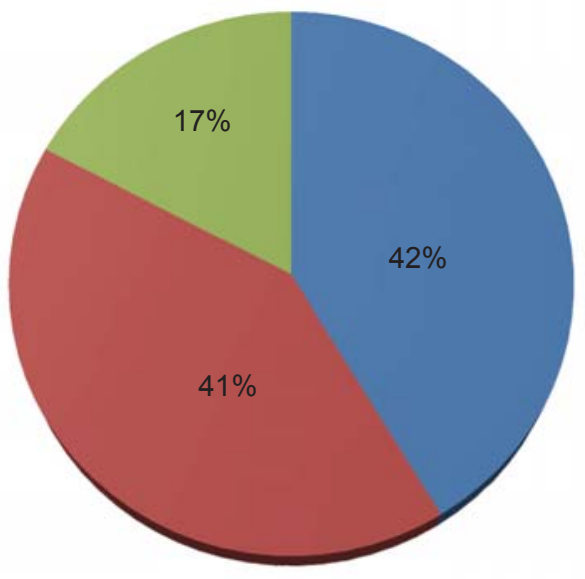

Streptococcus pneumoniae (12)

Hemophilus influenzae (12)

Group B Streptococcus (5)

Fig 1: Showing the causative organisms of acute bacterial meningitis.

\section{Discussion}

ABM in children is a potentially life threatening condition requiring early diagnosis, prompt identification of the causative organism and institution of effective therapy ${ }^{5}$. The yield of bacterial culture in our country is low, hence there was need to evaluate a test that could perform better and could rapidly identify the causative organism of ABM. Ours is the first study to evaluate LAT against bacterial culture in Nepal.

Streptococcus pneumoniae, Hemophilus influenza and Group B Streptococcus were the commonest causative organisms isolated in our study. This is in accordance to the organisms isolated from CSF in other studies $6,7,8,9$. Seasonal variation in the incidence of meningococcal meningitis and vaccination against it might have accounted for the fact that Neisseria meningitidis was not isolated in any of the CSF samples in our study. Of the $150 \mathrm{CSF}$ samples analysed culture could identify organism in only 4 and LAT in 29 giving an isolation rate of $1.3 \%$ and $19.3 \%$ respectively. The sensitivity of LAT $(60.5 \%)$ was higher than that of CSF culture $(10.5 \%)$. Similar results have been observed in other studies with sensitivity of LAT ranging from 60 to $95 \%$ and specificity from 70 to $98 \% \%^{10,11,12,13,14}$. However there have been conflicting results in the literature regarding the utility of LAT with studies suggesting that it is no better than using Gram's stain and CSF culture 
together ${ }^{15,16,17}$. These studies are mostly from countries where the yield of CSF culture is high as compared to our country.

One of the drawbacks of our study is the fact that we did not include Gram's stain in our study protocol. Secondly, in the definition of confirmed case only 4 had positive culture and the rest were classified as confirmed on the basis of CSF criteria's which are not the gold standard for the diagnosis of ABM and this might have affected the sensitivity and specificity of LAT. Thirdly, we did not look into how early diagnosis using LAT affected disease outcome in terms of mortality, reduction in the duration of hospital stay, cost of therapy and unnecessary use of empirical antibiotic therapy instead of specific therapy based on the organism identified. However this study forms a foundation on the basis of which further studies to look into cost effectiveness of using LAT can be performed. Study to see whether early detection of organism and subsequent specific therapy brings about a decrease in mortality and morbidity, duration of hospital stay, antibiotic resistance needs to be done.

The strength of our study is that in a country where the yield of culture is low and where culture is not readily available simple and rapid test like the LAT can be used in the peripheral set up to promptly identify the organism and start specific therapy. This becomes especially more important as steroids have a role in reducing mortality and morbidity in ABM due to Hemophilus influenza type $b$ if they are given for the first 2 days. Secondly, LAT can be extremely useful if there is an outbreak of ABM. It is yet to be studied whether the implementation of LAT in the peripheral hospitals will bring about an effect similar to that brought by the use of Rapid antigen capture test for Plasmodium falciparum malaria. Though the technology for detection of bacterial antigen in CSF by latex agglutination is still imperfect for widespread use, it still can have a major impact in a country like ours.

In conclusion as LAT has a better yield, provides microbiological diagnosis earlier than the traditional CSF culture and is easy to perform, it should be provided by the government as a part of national programme to the peripheral centres.

Acknowledgements: We would like to thank Dr. Rahul Chaudhary for the write up and editing. He is also grateful to all the staff of Kanti Children's Hospital.

Funding: Nil

Conflict of interest: This study is a part of the dissertation (thesis) submitted in 2006, during post graduate studies (MD) to National Academy of Medical Sciences (NAMS), Bir Hospital, Kathmandu, Nepal.

Permission from IRB: Yes

\section{References}

1. C H O R I [Internet]. [cited 2012 Sep 18]. Available from: http://www.chori.org/Principal_Investigators/ Granoff_Dan_M/granoff_publications.html

2. Peltola H. Worldwide Haemophilus influenzae type $\mathrm{b}$ disease at the beginning of the 21st century: global analysis of the disease burden 25 years after the use of the polysaccharide vaccine and a decade after the advent of conjugates. Clin. Microbiol Rev 2000;13(2):302-17.

3. Farag HFM, Abdel-Fattah MM, Youssri AM. Epidemiological, clinical and prognostic profile of acute bacterial meningitis among children in Alexandria, Egypt. Indian J Med Microbiol 2005;23(2):95-101.

4. El Bashir H, Laundy M, Booy R. Diagnosis and treatment of bacterial meningitis. Arch Dis Child 2003;88(7):615-20.

5. Ouédraogo SM, Yaméogo TM, Kyelem CG, Poda GEA, Ouédraogo NF, Millogo A, et al. Acute bacterial meningitis with soluble antigen detected by latex particle agglutination tests at the SourôSanou University Hospital of Bobo-Dioulasso (Burkina Faso). Med Sante Trop 2012;12(4);41216.

6. Vashishtha VM, Garg A, John TJ. Etiology of acute bacterial meningitis in hospitalized children in western Uttar Pradesh. Indian Pediatr 2011;48(12):985-6.

7. Mani R, Pradhan S, Nagarathna S, Wasiulla $\mathrm{R}$, Chandramuki A. Bacteriological profile of community acquired acute bacterial meningitis: a ten-year retrospective study in a tertiary neurocare centre in South India. Indian J Med Microbiol 2007;25(2):108-14.

8. Shameem S, Vinod Kumar CS, Neelagund YF. Bacterial meningitis: rapid diagnosis and microbial profile: a multicentered study. J Commun Dis 2008;40(2):111-20.

9. Narkeviciute I, Bernatoniene J, Mikelionyte A, Bernatoniene G, Baliukynaite V, Eidukevicius R. Aetiological diagnostics of acute bacterial meningitis in children. Scand. J Infect Dis 2006;38(9):782-7.

10. Berkley JA, Mwangi I, Ngetsa CJ, Mwarumba S, Lowe BS, Marsh K, et al. Diagnosis of acute bacterial meningitis in children at a district hospital in subSaharan Africa. Lancet 2001;357(9270):1753-7.

11. Abdel-Ghani SM, Hassan EM, Masoud S, Guirgis NI. Rapid diagnosis of bacterial meningitis by latex agglutination test. J Egypt Public Health Assoc 1989;64(1-2):31-44. 
12. Borel T, Rose AMC, Guillerm M, Sidikou F, Gerstl S, Djibo A, et al. High sensitivity and specificity of the Pastorex latex agglutination test for Neisseria meningitidis serogroup A during a clinical trial in Niger. Trans $R$ Soc Trop Med Hyg 2006;100(10):964-9.

13. Shivaprakash MR, Rajagopal V, Nagarathna S. Latex agglutination test in the diagnosis of pyogenic meningitis. J Commun Dis 2004;36(2):127-31.

14. Surinder K, Bineeta K, Megha M. Latex particle agglutination test as an adjunct to the diagnosis of bacterial meningitis. Indian J Med Microbiol 2007;25(4):395-7.
15. Barlow JF. Is BAT BAD in CSF? $S D J$ Med 1996;49(3):85.

16. Tarafdar K, Rao S, Recco RA, Zaman MM. Lack of sensitivity of the latex agglutination test to detect bacterial antigen in the cerebrospinal fluid of patients with culture-negative meningitis. Clin Infect Dis 2001;33(3):406-8.

17. Finlay FO, Witherow $\mathrm{H}$, Rudd PT. Latex agglutination testing in bacterial meningitis. Arch Dis Child 1995;73(2):160-1. 